\title{
Community Pharmacy Incident Reporting: A New Tool for Community Pharmacies in Canada
}

Certina Ho, Patricia Hung, Gary Lee and Medina Kadija

\begin{abstract}
Incident reporting offers insight into a variety of intricate processes in healthcare. However, it has been found that medication incidents are under reported in the community pharmacy setting.

The Community Pharmacy Incident Reporting (CPhIR) program was created by the Institute for Safe Medication Practices Canada specifically for incident reporting in the community pharmacy setting in Canada. The initial development of key elements for CPhIR included several focusgroup teleconferences with pharmacists from Ontario and Nova Scotia. Throughout the development and release of the CPhIR pilot, feedback from pharmacists and pharmacy technicians was constantly incorporated into the reporting program. After several rounds of iterative feedback, testing and consultation with community pharmacy practitioners, a final version of the CPhIR program, together with selfdirected training materials, is now ready to launch.

The CPhIR program provides users with a one-stop platform to report and record medication incidents, export data for customized analysis and view comparisons of individual and aggregate data. These unique functions allow for a detailed analysis of underlying contributing factors in medication incidents. A communication piece for pharmacies to share their experiences is in the process of development. To ensure the success of the CPhIR program, a patient safety culture must be established.
\end{abstract}

By gaining a deeper understanding of possible causes of medication incidents, community pharmacies can implement system-based strategies for quality improvement and to prevent potential errors from occurring again in the future. This article highlights key features of the CPhIR program that will assist community pharmacies to improve their drug distribution system and, ultimately, enhance patient safety.

$\mathrm{P}$ atient safety has become an increasingly significant aspect of healthcare. One method to improve patient safety is to learn from breakdowns in the healthcare system that lead to potential harm to patients (World Health Organization [WHO] 2005). To learn from these failures, these incidents need to be brought to light and reported. Incident reporting offers insight into a variety of intricate processes in healthcare. An incident typically occurs after multiple factors fail in a cascade of interconnected events, rather than a single factor at one point during the delivery of care to the patient. By reporting incidents, healthcare practitioners are able to investigate the root causes of the incident and learn by making changes in the system to prevent a future occurrence.

While other healthcare settings have an increased awareness about patient safety, community pharmacy seems to lag behind (MacKinnon 2006). In certain healthcare settings, including hospitals and long-term care facilities, a reporting system is a required organizational practice by Accreditation Canada, a national standard-setting organization (Accreditation Canada 
2008). However, standards in community pharmacy are set by each individual provincial regulatory body, so community pharmacies throughout Canada may have different practices regarding incident reporting. Although there is a national reporting system, the Individual Practitioner Reporting by the Institute for Safe Medication Practices Canada (ISMP Canada; available at https://www.ismp-canada.org/err_report. $\mathrm{htm}$ ), where any healthcare practitioner can report medication incidents, it has been found that medication incidents are underreported in the community pharmacy setting relative to other institutions (Cheng et al. 2010). This lack of reporting may be due to the uniqueness of the community pharmacy setting compared with other settings. In contrast to other healthcare institutions, the majority of patients at a community pharmacy are ambulatory and do not require medication administration or direct medical assistance. The main purpose of community pharmacies is to distribute medications and provide pharmaceutical care for patients. As most reporting systems available cater to hospitals or long-term care facilities, there is a need for an incident reporting system specific to the community pharmacy setting that allows the study of factors or work processes in a community/outpatient medication-distribution system.

Currently, some of the larger corporate pharmacies do have a reporting system in place. However, these systems are typically used for legal purposes. When a medication incident occurs, the pharmacist is required to report the incident to mitigate any liability. These systems are usually not anonymous, so pharmacists may feel intimidated to report incidents for fear of being reprimanded. Furthermore, these existing systems are rarely implemented to understand contributing factors of medication incidents, so many of the reported medication incidents at one location can potentially occur again at another location of the corporate pharmacy.

ISMP Canada developed the Community Pharmacy Incident Reporting (CPhIR) program, the first national incident reporting program made specifically for community pharmacies. This article discusses the development and highlights the key features of the CPhIR program that will assist community pharmacies to improve their drug distribution system and, ultimately, enhance patient safety.

\section{Development}

As a starting point for the development of a reporting program for community pharmacy, the data elements from ISMP Canada's Individual Practitioner Reporting form were used. These elements, listed in Table 1, were presented to a research team, SafetyNET-Rx (http://www.safetynetrx.ca), in Nova Scotia. Nova Scotia has recently passed new quality assurance standards, which include the need to report near misses and medication incidents (or quality related events) in community pharmacies. ISMP Canada worked collaboratively with this research team to develop the initial key elements of the $\mathrm{CPhIR}$ program. The research team consisted of many stakeholders including community pharmacists, pharmacy technicians, members from the provincial regulatory body and researchers from academic institutions. After iterative discussion and teleconferences, data elements were finally customized for community pharmacy incident reporting. The elements from Table 1 were then narrowed down to those listed in Table 2 for the CPhIR program. Most data elements removed were typically used in an acute care setting and were hence irrelevant to community pharmacy incident reporting.

After several months of development, the CPhIR program was released as a pilot project. Thirteen community pharmacies that had participated in the SafetyNET-Rx phase I pilot project in 2008 were invited to test the CPhIR program by submitting mock-up medication incidents to the training/ demonstration site of CPhIR (http://www.cphir.ca/training). Several Ontario pharmacies also had the opportunity to view and pilot-test the CPhIR program at the same time, including independent, grocery, mass merchandising and chain pharmacies. They provided feedback to the CPhIR development team and were invited to participate in future teleconferences.

In July 2009, two focus groups via teleconference, one from Ontario and one from Nova Scotia, were invited to test the reporting feature of the program (Figure 1). Individuals in these focus groups included pharmacists, managers from the corporate office of chain pharmacies, members of provincial pharmacy associations and researchers from academic institutions. Modifications of the Report an Incident interface took place based on recommendations from the focus group participants.

Subsequent teleconferences were arranged with pharmacy practitioners in Ontario and Nova Scotia in August 2009, December 2009 and March 2010, seeking their feedback and input to the Search, Stats and Account Management components of the CPhIR program, respectively (Figures $2-5$ ). While the Search and Account Management components were released at once with minor feedback, the Stats function was released in four phases. The four phases included downloading the statistics within CPhIR to the user's local computer hard drive, exporting individual statistics into Microsoft Office Excel for internal and customized analysis, comparing individual and aggregate data in frequency tables within CPhIR and, finally, comparing individual and aggregate data in graphs within CPhIR. As each phase was released, feedback from pharmacists and pharmacy technicians was incorporated.

CPhIR continues to receive minor updates based on feedback from users of the program after they have tested the reporting system through the submission of mock-up medication incidents to the CPhIR training/demonstration website. Once all final updates are completed, ISMP Canada plans to allow users to become comfortable with the final product for a 
Table 1. Individual practitioner reporting core data set

\begin{tabular}{|c|c|c|c|}
\hline \multicolumn{2}{|c|}{ Individual Practitioner Reporting Data Elements } & \multirow{2}{*}{$\begin{array}{l}\text { Mandatory/Optional Indicator } \\
\text { Optional }\end{array}$} & \multirow{2}{*}{$\begin{array}{l}\text { Input Type } \\
\text { Pop-up calendar }\end{array}$} \\
\hline Incident & Date of incident & & \\
\hline & Time of incident & Optional & Text box \\
\hline & Incident description/how discovered & Mandatory & Text box \\
\hline & Stages involved & Mandatory & Check boxes \\
\hline & Type of incident & Mandatory & Pull-down menu \\
\hline & Discovered by & Mandatory & Pull-down menu \\
\hline & Care area type & Mandatory & Pull-down menu \\
\hline \multirow[t]{2}{*}{ Outcome } & Severity/outcome & Mandatory & Radio buttons \\
\hline & Intervention & Optional & Text box \\
\hline \multirow[t]{7}{*}{ Medication(s) } & Medication name & Mandatory & Text box \\
\hline & Strength & Optional & Text box \\
\hline & Route of administration & Optional & Text box \\
\hline & Manufacturer & Optional & Text box \\
\hline & Lot number & Optional & Text box \\
\hline & Confusing drug name, label or packaging & Mandatory & Radio buttons \\
\hline & Upload picture or PDF file & Optional & File input \\
\hline \multirow[t]{3}{*}{ Follow-Up } & Action & Optional & Text box \\
\hline & Comments/recommendations & Optional & Text box \\
\hline & System improvement strategies implemented & Optional & Text box \\
\hline \multirow[t]{3}{*}{ Patient } & Non-patient specific & Optional & Check box \\
\hline & Age category & Optional & Pull-down menu \\
\hline & Gender & Optional & Radio buttons \\
\hline \multirow[t]{7}{*}{ Reporter } & Name & Optional & Text box \\
\hline & Practice setting & Optional & Text box \\
\hline & City & Optional & Text box \\
\hline & Province & Optional & Pull-down menu \\
\hline & Postal code & Optional & Text box \\
\hline & Email & Optional & Text box \\
\hline & Permission to contact reporter & Optional & Text box \\
\hline
\end{tabular}




\begin{tabular}{|l|l|l|l|}
\hline Individual Practitioner Reporting Data Elements & Mandatory/Optional Indicator & Input Type \\
\hline $\begin{array}{l}\text { Possible contributing } \\
\text { factors }\end{array}$ & Critical patient information missing & Optional & Select menu \\
\cline { 2 - 4 } & Critical drug information missing & Optional & Select menu \\
\cline { 2 - 4 } & Miscommunication of drug order & Optional & Select menu \\
\cline { 2 - 4 } & Drug name, label or packaging problem & Optional & Select menu \\
\hline & Drug storage or delivery problem & Optional & Select menu \\
\cline { 2 - 4 } & Drug delivery device problem & Optional & Select menu \\
\cline { 2 - 4 } & Environmental, staffing or workflow problem & Optional & Select menu \\
\cline { 2 - 4 } & Staff education problem & Optional & Select menu \\
\cline { 2 - 4 } & Patient education problem & Optional & Select menu \\
\cline { 2 - 4 } & Lack of quality control or independent check systems & Optional & Select menu \\
\hline
\end{tabular}

PDF = portable document format.

Source: Adapted from Canadian Medication Incident Reporting and Prevention System Core Data Set for Individual Practitioner Reporting, available at http://www.ismp-canada.org/cmirps.htm).

Table 2. CPhIR Core Data Set

\begin{tabular}{|c|c|c|}
\hline CPhIR Data Elements & $\begin{array}{l}\text { Mandatory/ } \\
\text { Optional Indicator }\end{array}$ & Input Type \\
\hline Date incident occurred & Mandatory & Calendar \\
\hline Time incident occurred & Optional & Pull-down menu \\
\hline Type of incident & Mandatory & Radio buttons \\
\hline Incident discovered by & Mandatory & Pull-down menu \\
\hline Medication system stages involved in this incident & Mandatory & Check boxes \\
\hline Medications & Mandatory & Text box \\
\hline Patient's gender & Optional & Pull-down menu \\
\hline Patient's age & Optional & Pull-down menu \\
\hline Degree of harm to patient due to incident & Mandatory & Radio buttons \\
\hline Incident description/how incident was discovered & Mandatory & Text box \\
\hline Other incident info & Optional & Check boxes \\
\hline Contributing factors of this incident & Optional & Check boxes \\
\hline Actions at store level (Include action plan, person in charge, and target date for completion) & Optional & Text box \\
\hline $\begin{array}{l}\text { Shared learning for ISMP Canada to disseminate (What has been done to prevent a similar occurrence in } \\
\text { the future) }\end{array}$ & Optional & Text box \\
\hline
\end{tabular}

CPhIR = Community Pharmacy Incident Reporting; ISMP Canada = Institute for Safe Medication Practices Canada. 
Figure 1. Reporting feature of the CPhIR program

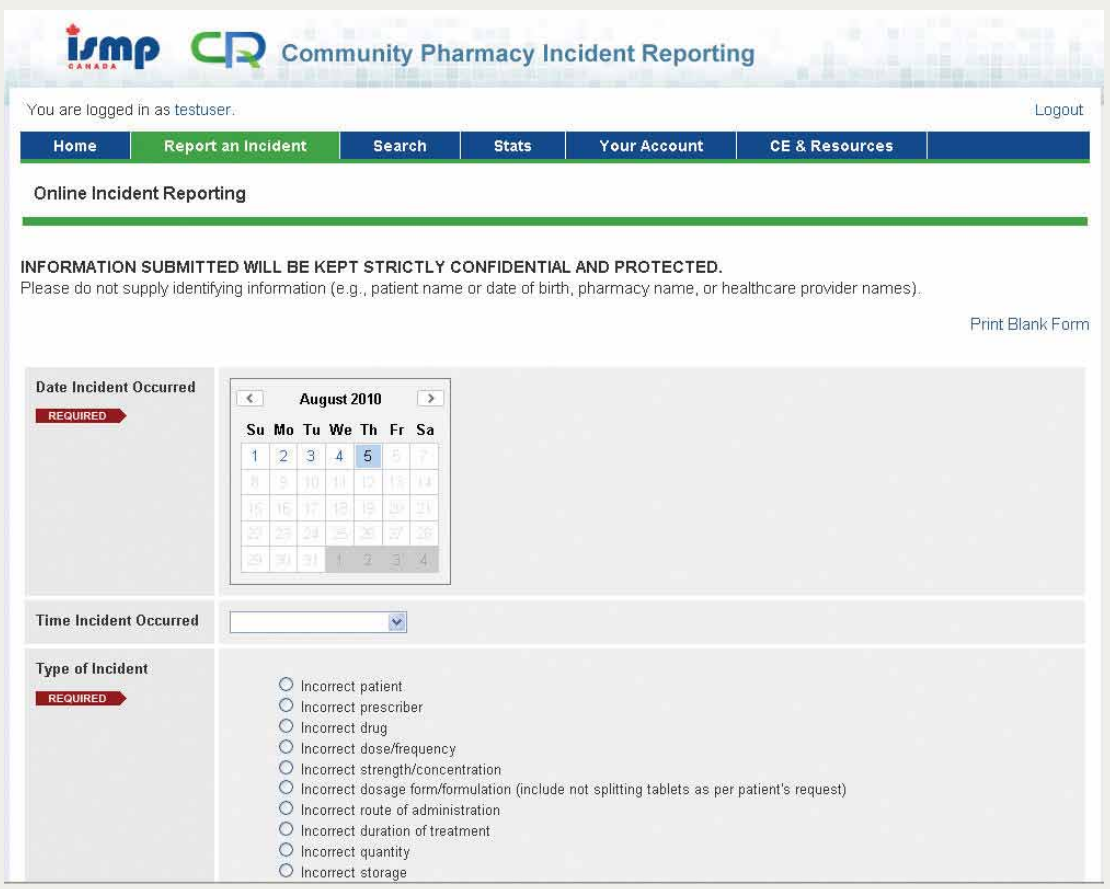

Figure 2. Search feature of the CPhIR program

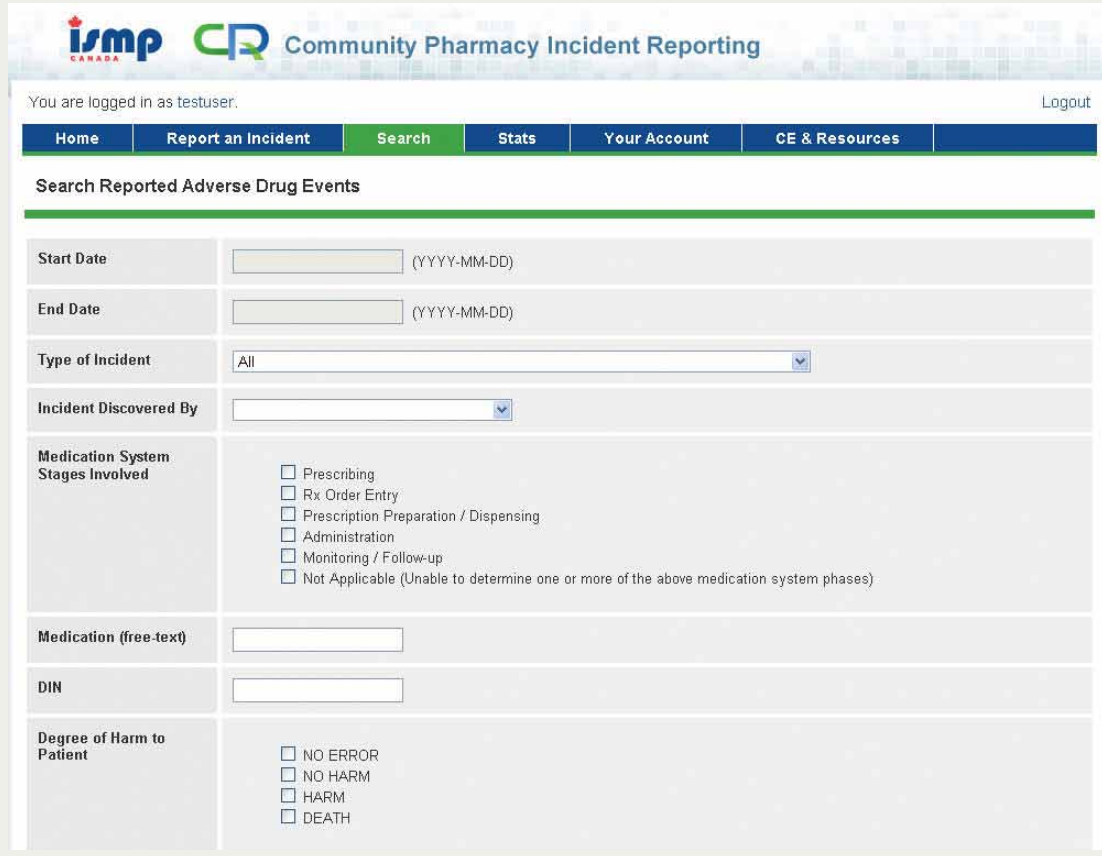

many users from the 13 community pharmacies in Nova Scotia and the pharmacies in Ontario have deemed the program easy to use and have said that, in general, they are able to complete the data entry and submission of an incident report within approximately five minutes. In fact, the user-friendliness of the CPhIR program has allowed some of these users to switch from employing a paper-based reporting form to directly inputting the incident information online at the CPhIR website upon the occurrence and discovery of an incident.

\section{Implementation}

As of April 2010, the CPhIR program is available to community pharmacies at http://www.cphir. ca. An annual subscription fee is required for $\mathrm{CPhIR}$ that includes the use of the program, electronic access of ISMP Canada Safety Bulletins, SafeMedicationUse.ca Newsletter, and Medication Safety Alerts throughout the year. Since CPhIR is easily accessible from any location with an Internet connection, pharmacies in rural locations are also able to use the program. CPhIR is a one-stop platform with the following components - Report an Incident (see Figure 1), Search (see Figure 2), Stats (see Figures 3 and 4), Your Account (see Figure 5) and CE \& Resources (Figure 6). Frequently asked questions (FAQs) (Figure 7) are also available online.

\section{Registration}

The registration function is an internal function used by ISMP Canada to register users. To complete registration, the pharmacy must complete a data sharing agreement, which states that the pharmacy agrees to share information regarding few months before completing a formal extensive evaluation of the program. Although no formal evaluation has taken place, reported medication incidents with ISMP Canada. The registration is completed by one designated employee at ISMP Canada 
Figure 3. Statistics feature of the CPhIR program

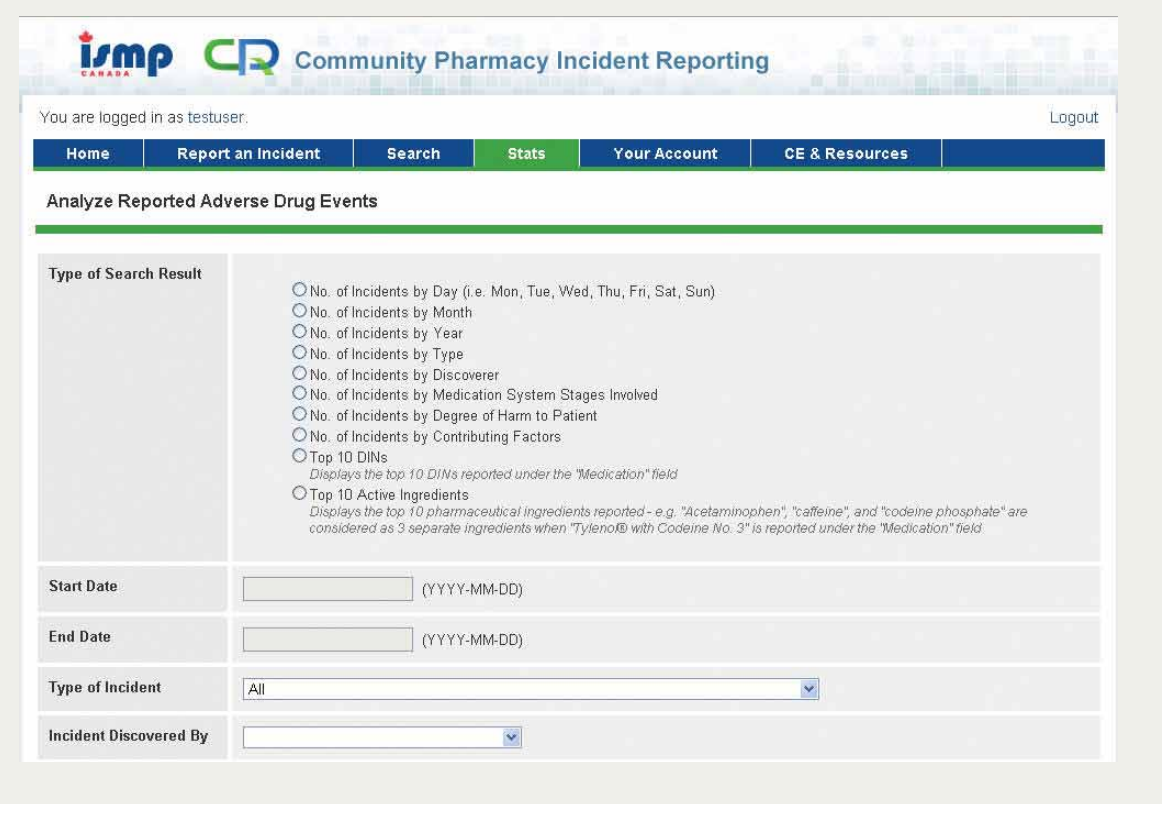

Figure 4. Statistics feature of the CPhIR program - incidents by day
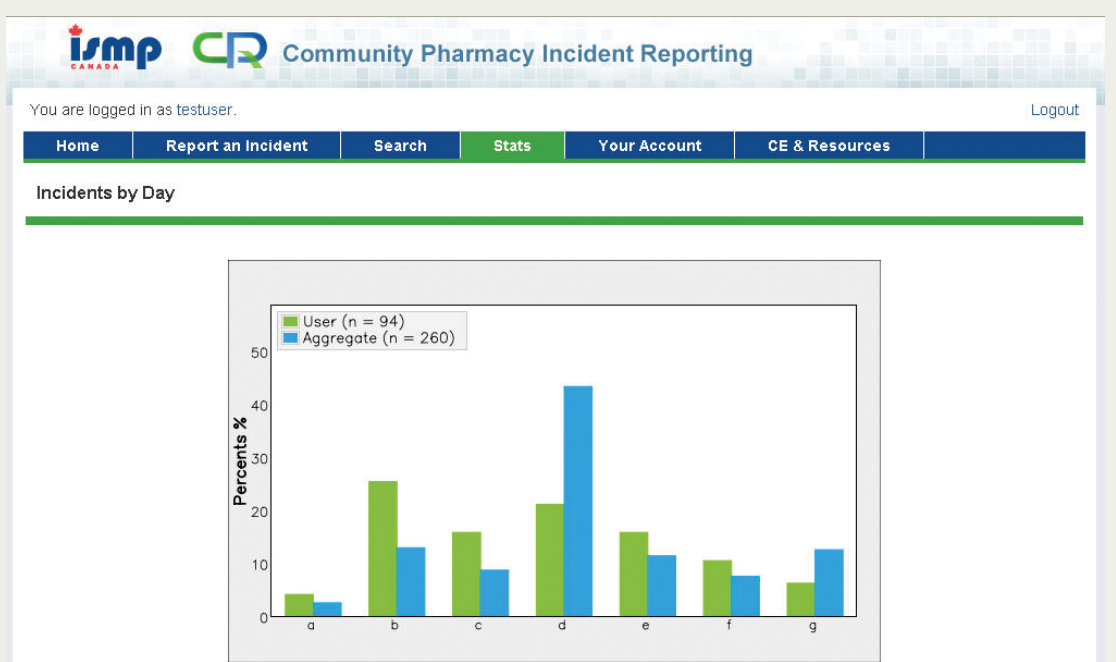

\begin{tabular}{|l|r|r|}
\hline User Values & $\#$ & \\
\hline a- Sunday & 4 & 4 \\
\hline b- Monday & 24 & 26 \\
\hline c- Tuesday & 15 & 16 \\
\hline d - Wednesday & 20 & 21 \\
\hline e- Thursday & 15 & 16 \\
\hline f- Friday & 10 & 11 \\
\hline g- Saturday & 6 & 6 \\
\hline
\end{tabular}

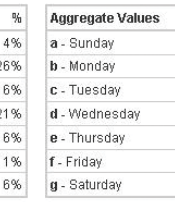

\% Saturday incidents reported from pharmacies essentially remain anonymous.

\section{Home}

Currently, the home page of CPhIR lists open incidents as a reminder for users to close and submit these reports after they login. An open incident is similar to a draft report where the user has entered some information but has not submitted a final copy to ISMP Canada. This allows users to start entering information when an incident is discovered but to have more time to collect further details of the incident before entering all the information. Open incidents can be edited up to 90 days before they are automatically submitted to the ISMP Canada national incident database.

\section{Report an Incident}

Table 2 displays the fields in the reporting interface of $\mathrm{CPhIR}$. With a combination of check boxes, pulldown menus and radio buttons for selection, the form is relatively easy to use. Since the reporting process is not likely to be time consuming, pharmacy staff members are encouraged to fill out the form for both near misses and medication incidents upon the occurrence or discovery of the event.

\section{Search}

The Search function allows users to retrieve an individual or a series of incidents based on self-determined criteria. When the user retrieves a cluster of incidents, these can be exported to Microsoft Office Excel $^{\circledR}$. Once the data are exported, the user can then perform a customized analysis for the individual pharmacy. The user can employ

who does not have access to reported medication incidents. This individual assigns the pharmacy a unique username, which is only accessible by this individual. By delegating different tasks involved with CPhIR to different employees, medication descriptive statistics to provide an analysis of any mandatory data elements listed in Table 2. This feature is particularly useful for pharmacies to analyze reported incidents and to determine possible contributing factors of these incidents. Once the causes 
Figure 5. Account management of the CPhIR program

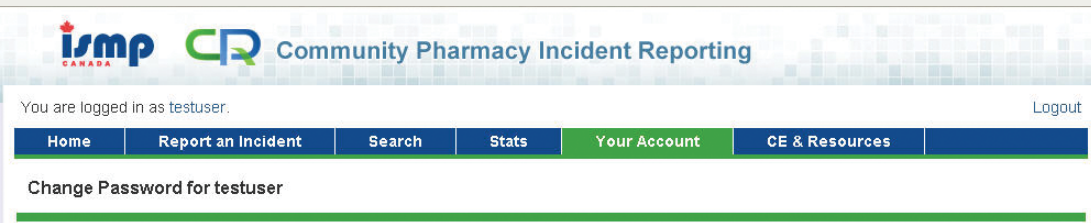

Do not use the same password that you use for other online accounts. - Your new password must be at least 8 characters in length. - Use a combination of letters, numbers, and punctuation.

Passwords are case-sensitive. Remember to check your CAPS lock key.

Old password:

New password:

Re-enter your new password:

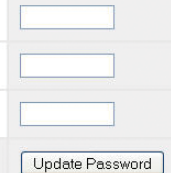

Update Password

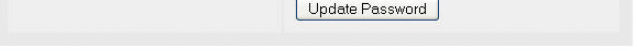

Your Account Activity:

Incidents Reported

Open Incidents

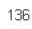

Records will bo
leporting.)

Clear Remembered Sessions

If, for security reasons, you wish to clear all "Remember

Me" sessions for your account, click the button below:

Clear Remembered Sessions

This will force every browser to re-login to CPhIR.

\section{Home | Report | Search | FAQ}

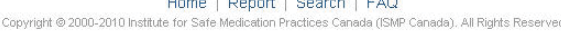

Figure 6. CPhIR CE \& Resources (CE = Continuing Education) centre

the Community Pharmacy Incident Reporting

You are logged in as testuser

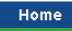

Search

Stats

Your Account

CE Modules

\begin{tabular}{|l|l|l|l|l|l|}
\hline Modules & Title & Speakers & Date & Duration & Slides \\
\hline 1 & Medication Safety $101^{*}$ & $\begin{array}{l}\text { Roger Cheng, RPh, BScPhm, PharmD } \\
\text { Patricia Hung. B.Sc.Phm. Candidate }\end{array}$ & April 2010 & $30: 31$ & 37 Slides \\
\hline 2 & $\begin{array}{l}\text { CPhlR Training Guide } \\
\text { Canadian Medication Incident } \\
\text { Reporting and Analysis }\end{array}$ & Patricia Hung, B.Sc.Phm. Candidate & April 2010 & $61: 22$ & 50 Slides \\
\hline 3 & $\begin{array}{l}\text { Solution Development } \\
\text { Examining Possible Interventions }\end{array}$ & Calver Cheng. RPh, BscPhm, PharmD & April 2010 & $45: 10$ & 36 Slides \\
\hline 4 & Coon, B.Sc.Phm. Candidate & August 2010 & $24: 02$ & 24 Slides \\
\hline
\end{tabular}

* This training module has been accredited by the Ontario College of Pharmacists
This function compares the statistics of medication incidents at the individual pharmacy level versus a national aggregate of all incidents reported through CPhIR from community pharmacies across Canada. This is valuable because users are able to identify trends and whether certain incidents are specific to a particular pharmacy setting or are generalized to all community pharmacies across the nation.

\section{CE \& Resources}

The CE \& Resources centre includes modules about patient safety. The first module provides an overview of medication safety consisting of the significance of medication incidents, human and environmental factors and the system approach to medication safety. This module should educate users about the importance of medication safety and help to shape an open culture toward reporting medication incidents for the purpose of shared learning. The second module is a tutorial that teaches users how to use the CPhIR program, including all functions described above. A third module provides a brief description on different methods to conduct meaningful medication incident analyses. A fourth module offers various error reduction strategies or solution development after identification of causes or contributing factors of medication incidents in a practice setting. Further CE training modules will be available as CPhIR continues to develop.

of medication incidents have been uncovered, system-based strategies can be implemented in the work environment to prevent the reoccurrence of similar events.

\section{Statistics}

Although certain contributing factors can only be associated to an individual community pharmacy, other factors may be present in the distribution systems of all community pharmacies in general. The Stats function addresses this concern.
All reported near misses and medication incidents will be submitted to the ISMP Canada national incident database, which contributes to the Canadian Medication Incident Reporting and Prevention System (http://www.ismp-canada. org/cmirps.htm). ISMP Canada will analyze the medication incidents and provide recommendations for medication safety and continuous quality improvement in community pharmacy practice via the dissemination of safety bulletins or newsletters. 
Figure 7. Frequently asked questions regarding the CPhIR program

InP Community Pharmacy Incident Reporting

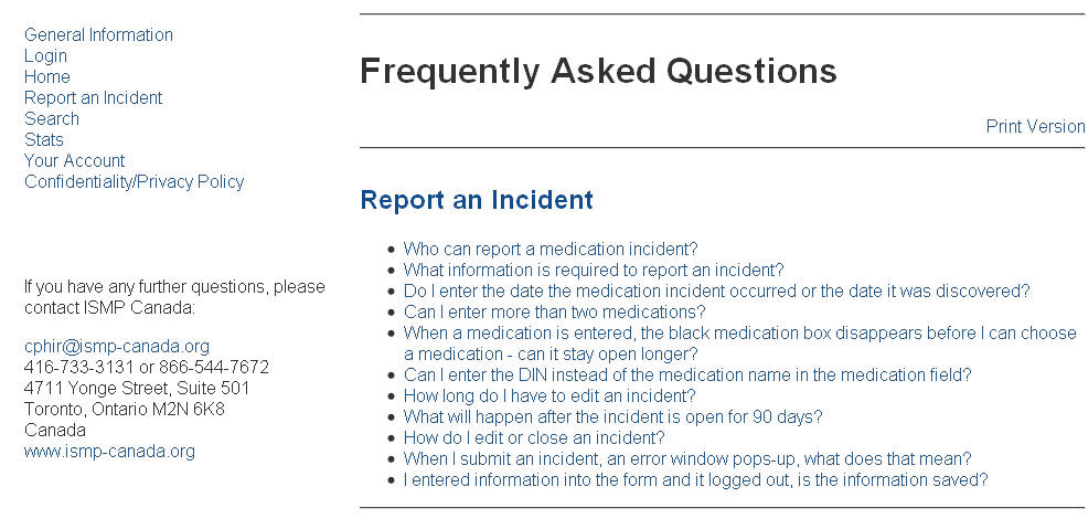

Who can report a medication incident?

- The fundamental role of patient safety reporting systems is to enhance patient safety by learning from failures of the health-care system.

- Reporting must be safe. Individuals who report incidents must not be punished or suffer other ill-effects from reporting.

- Reporting is only of value if it leads to a constructive response. At a minimum, this entails feedback of findings from data analysis. Ideally, it also includes recommendations for changes in processes and systems of healthcare.

- Meaningful analysis, learning, and dissemination of lessons learned require expertise and other human and financial resources. The agency

Quarterly updates are planned to be released to all CPhIR users providing information about medication incidents reported through CPhIR.

\section{Next Steps}

As more medication incidents are reported to ISMP Canada via the CPhIR Program, ISMP Canada intends to introduce a communication platform to CPhIR. Preliminary plans include weekly tips and quarterly newsletters regarding medication safety through learning from incidents submitted to CPhIR. By offering a communication channel, ISMP Canada would like to encourage open dialogue and shared learning among all community pharmacies in Canada for the common goal of enhancing patient safety.

To ensure the success of the program, a culture toward patient safety must be established in community pharmacy practices. Ashcroft et al. (2005) commented that pharmacists and pharmacy technicians do not report incidents because they feel the risks of being blamed outweigh the benefits of learning from the incident. Therefore, it is essential to establish an open culture where medications incidents are freely discussed and a system-based strategy is the focal point of discussion to learn, rather than a blame-and-shame approach, which is ineffective and meaningless toward patient safety.

\section{Conclusion}

The WHO Draft Guidelines for Adverse Event Reporting and Learning Systems describes four core concepts of a patient safety reporting system: that receives reports must be capable of disseminating information, making recommendations for changes, and informing the development of solutions. (2005: 10)

The CPhIR program aligns with the above core concepts. CPhIR was built to learn from failures in the system processes of community pharmacies. It is a special program made to accommodate the unique setting of the community pharmacy. The second concept states that reporters must feel safe using a reporting program. In the CPhIR program, (1) data are transmitted to ISMP Canada securely and anonymously so that no blame can be associated to the reporter(s) at the community pharmacy; and (2) a no-blaming culture is encouraged internally at the community pharmacy so that no individual staff member is punished. The WHO guidelines suggest that a meaningful analysis must be completed to understand how errors occur and what recommendations can be made to improve the system. CPhIR includes features that can assist individual community pharmacy in these analyses by allowing the user to generate descriptive statistics (such as frequency tables and graphs) on the mandatory data elements listed in Table 2 via the Stats function (see Figure 4) when users login with their unique username and password. Finally, the "agency" referred in the WHO guidelines is ISMP Canada. All reported medication incidents will be transmitted to the ISMP Canada national incident database, where experts in the medication safety field can analyze the incidents, make recommendations and disseminate findings and learning to healthcare practitioners in Canada. The ISMP Canada Safety Bulletins (available at http://www.ismp-canada.org/ISMPCSafetyBulletins. 
$\mathrm{htm}$ ) and the anticipated communication platform in CPhIR will be the means to fulfill the notion of the dissemination of recommendations and development of solutions.

$\mathrm{CPhIR}$ is a brand new tool that will change the state of incident reporting in community pharmacies. By gaining a deeper understanding of possible causes of medication incidents, community pharmacies can implement system-based strategies for quality improvement and the prevention of potential errors from occurring again in the future, which will ultimately enhance patient safety. HQ

\section{Acknowledgements}

ISMP Canada would like to acknowledge the support from the Ontario Ministry of Health and Long-Term Care for the development of the CPhIR program. The feedback from pharmacists and pharmacy technicians in Ontario and Nova Scotia (SafetyNET-Rx in Nova Scotia), funded by the Social Sciences and Humanities Research Council of Canada phase I pilot project 2008-2009), has been extremely helpful and is very much appreciated. CPhIR contributes to the Canadian Medication Incident Reporting and Prevention System.

\section{References}

Accreditation Canada. 2008. Patient Safety Area 1: Culture. Ottawa, ON: Author. Retrieved April 7, 2010. <http://www.accreditation.ca/uploadedFiles/Reporting\%20System.pdf?n=106>.

Ashcroft, D.M., C. Morecroft, D. Parker and P.R. Noyce. 2005. "Safety Culture Assessment in Community Pharmacy: Development, Face Validity, and Feasibility of the Manchester Patient Safety Assessment Framework.” Quality and Safety in Health Care 14: 417-21.

Cheng, R., C. Ho, C. Lee, S. von Guttenberg and L. Yoo. 2010. "Analysis of Medication Incidents in Ontario." Canadian Journal of Hospital Pharmacy 63(1): 68.

MacKinnon, N.J. 2006. "Is Community Pharmacy Falling Behind in the Patient Safety Movement?" Canadian Pharmacists Journal 139(5): 23-24.

World Health Organization. 2005. WHO Draft Guidelines for Adverse Event Reporting and Learning Systems. Geneva, Switzerland: Author. Retrieved March 16, 2010. <http://www. who.int/patientsafety/events/05/Reporting Guidelines.pdf>.

\section{About the Authors}

Certina Ho, BScPhm, MISt, MEd, is a project manager at the Institute for Safe Medication Practices Canada (ISMP Canada) and adjunct assistant professor at the School of Pharmacy, University of Waterloo, in Waterloo, Ontario. You can contact Certina Ho by email at cho@ismp-canada.org.

Patricia Hung, BScPhm candidate, is a pharmacy student at the School of Pharmacy, University of Waterloo, Waterloo, Ontario.

Gary Lee, BEng, is the information technology manager at ISMP Canada.

Medina Kadija, BA, is the administrative assistant at ISMP Canada.

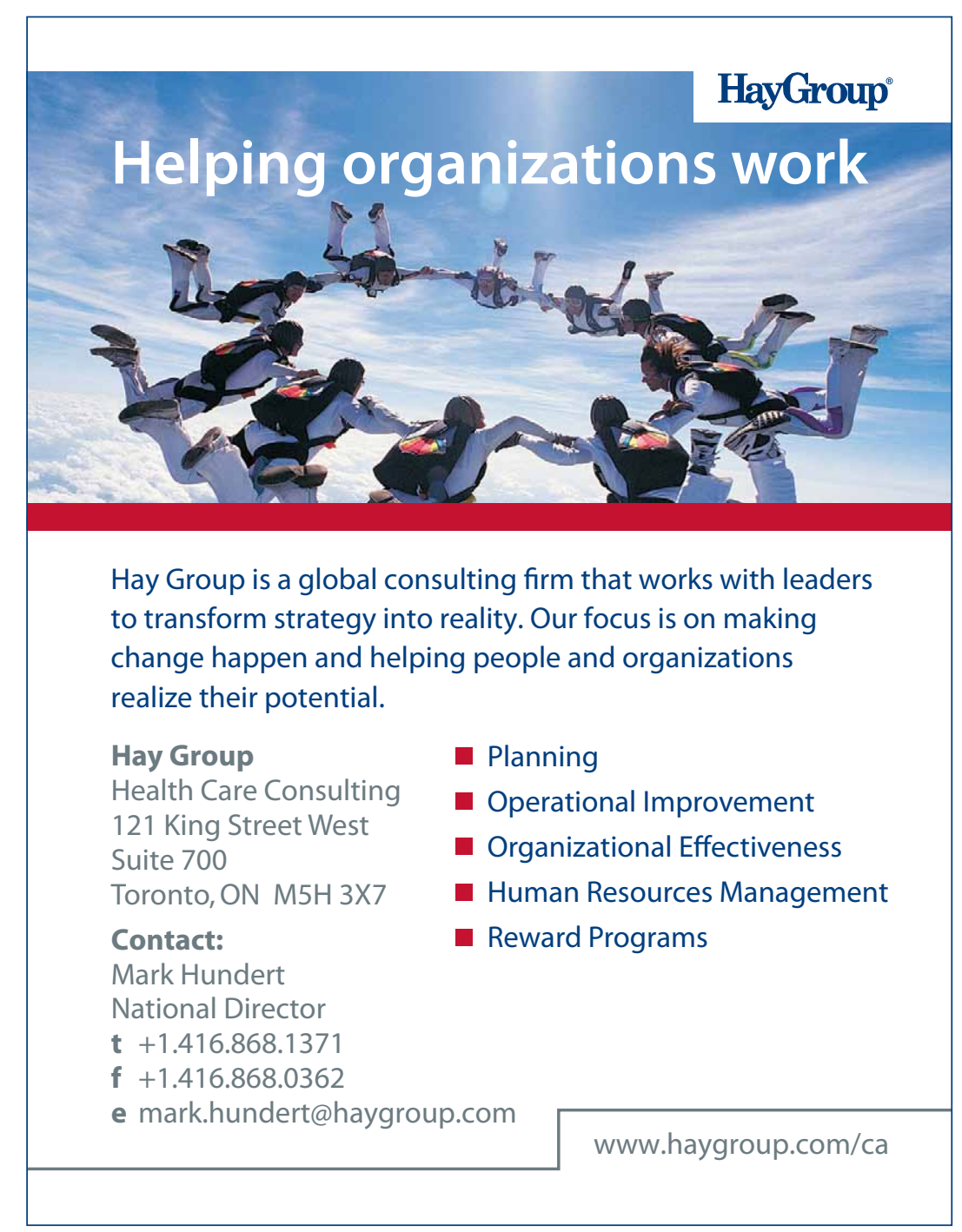




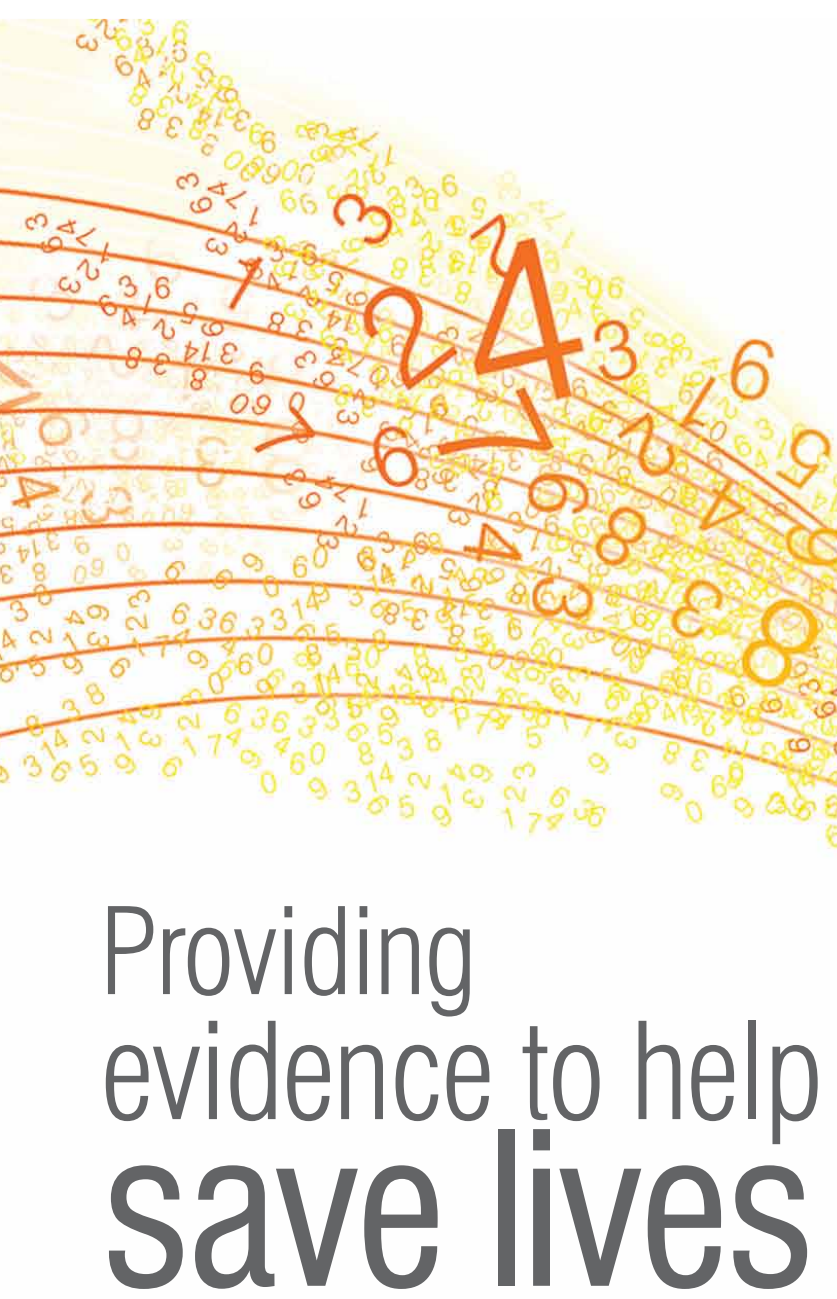

When reviewing HSMR data over the past five years, Southlake Regional Health Centre identified sepsis, a condition resulting from the body's response to severe infection, as a cause of death requiring further investigation. Recognizing the high mortality rates associated with sepsis nationally - three times as high as for heart attack patients - the centre is now focused on early identification and treatment of the condition and using the HSMR to monitor improvements over time. 